\section{e0145 STUDY OF MRI IN TRACKING MAGNETICALLY LABELLED MESENCHYMAL STEM CELLS}

doi:10.1136/hrt.2010.208967.145

Liu Qiong, Zhao Shihua, Jiang Shiliang, Lu Minjie, Ling Jian, Zhan Yan, Yan Chaowu, Cheng Huaibing, Ma Ning, Li Shiguo, Yin Gang. Department of Radiology, Cardiovascular Institute and Fuwai Hospital, Chinese Academy of Medical Sciences, Peking Union Medical College, Beijing, China

Objective To investigate the potential ability of MRI in tracking magnetically labelled mesenchymal stem cells (MR-MSCs) in a swine myocardial infarction (MI) model.

Methods Adult Chinese mini pigs $(n=6)$ were subjected to openchest experimental MI. Their autogeneic bone marrow-derived mesenchymal stem cells (MSCs) was cultured and doubly labled with ferumoxides and DAPI. At the 14th day after MI, labelled MSCs were injected intramyocardially into peri-infarct zone and normal myocardium. At the 14th day after MSCs transplantation, the size and location of the myocardial infarction were assessed by using delayed-enhancement MRI (DE-MRI). The contrast and the volume of the MR-MSCs hypointense lesion from the FGRE images were acquired. At 24 and 3 weeks after injection, the contrast was determined using the difference in signal intensity between the hypointense and normal myocardium divided by signal intensity of the normal region. After humane euthanasia, the heart was excised and histology corresponding to MRI slices that demonstrated MRMSCs lesions was performed.

Results At the 14th day after MSCs transplantation, DE-MRI showed the infarct in all animals and the mean infarction size was $(33.60 \pm 9.80) \%$ of the left ventricular area. At $24 \mathrm{~h}$ after injection, the contrast and the size of the lesions showed no statistical difference between in peri-infarct zone and in normal myocardium. At 3 weeks after injection, the contrast of the lesions in peri-infarct zone decreased rapidly than that in normal myocardium $(26.88 \pm 7.27$ vs $15.00 \pm 4.51, \mathrm{p}=0.0003)$. Post mortem analysis showed that fluorescently labelled MSCs were demonstrated on histological sections. There were much more dense fluorescently labled MSCs per high power fields in injection sites of normal myocardium than in injection sites of peri-infarct zone $(106 \pm 25 / \mathrm{HPF}$ vs $143 \pm 31 / \mathrm{HPF}$, $p=0.0293$ ). At 3 weeks, there was less fibrosis in MSCs injection sites with more surviving myocardium. In MSCs injection sites of the periinfarct zone, the capillary density was significantly more than that in control sites (13.40 $\pm 4.00 / \mathrm{HPF}$ vs $9.40 \pm 3.10 / \mathrm{HPF}, \mathrm{p}=0.0229)$.

Conclusions MRI of MSCs is feasible and represents a method for noninvasively tracking the quantity and location of intramyocardial delivery after MI. But to semi-quantitatively analyse the development of transplanted MSCs by MRI is not dependable.

\section{e0146 ALTERED EXPRESSION OF ADAMTS-1 AND ITS INHIBITOR IN RATS WITH CHRONIC HEART FAILURE INDUCED BY ADRIAMYCIN}

doi:10.1136/hrt.2010.208967.146

Li Jianqiang, Li Weimin, Li Yue, Zhao Jiyi, Kong Yihui, Guo Hong, Xue Jingyi, Sheng Li, Gong Yongtai, Xue Hongjie. The First Affiliated Hospital of Harbin Medical University, Harbin, China

Purpose The clinical progression of chronic heart failure (CHF) is largely determined by ventricular remodelling, which involves cellular and extracellular matrix disruption. A disintegrin and metalloproteinase with thrombospondin motifs- 1 (ADAMTS-1) is known to regulate cell-cell and cell-matrix interactions, and may thereby influence cardiac structure. The present study was undertaken to investigate the expression of ADAMTS-1 and its endogenous inhibitor, tissue inhibitor of metalloproteinases-3 (TIMP-3), in rats with CHF.
Methods 60 healthy male Wistar rats were randomly divided into a control group $(n=30)$ and a CHF group $(n=30)$. Adriamycin was administered intraperitoneally $(2.5 \mathrm{mg} / \mathrm{kg}$ each week) to rats in the CHF group for 6 weeks. Rats in the control group received saline in the same regimen with adriamycin treatment. Animals in both groups were observed for 4 weeks after the last injection for general appearance, behaviour, and mortality. Before and 10 weeks after initiating the study, body weight (BW), left ventricular weight (LVW), and LVW/ BW were measured; left ventricular end diastolic diameter (LVEDD), left ventricular ejection fraction (LVEF), and fractional shortening (FS) were assessed; and left ventricular systolic pressure (LVSP), left ventricular end diastolic pressure (LVEDP), and maximum rates of rise and fall of left ventricular pressure $\left( \pm d p / d t_{\max }\right)$ were recorded. 10 weeks after the study started, pathohistological and ultrastructural changes in ventricular tissue were examined by light and electron microscopy. Collagen volume fraction (CVF) and apoptotic index were analysed by Masson staining and TUNEL assay, respectively. Levels of ADAMTS-1, Syndecan-4, and TIMP-3 were evaluated using immunohistochemistry and western blot, respectively.

Results After the adriamycin treatment, BW in the CHF group was slowly increased, while LVW was quickly increased, resulting in a higher LVW/BW than that in the control group $(p<0.01)$. Compared with the control group, LVEF, FS, LVSP, and $\pm \mathrm{dP} / \mathrm{dt}_{\max }$ were reduced in the CHF group $(p<0.05)$, whereas LVEDD and LVEDP were augmented $(p<0.01)$. There was a significant increase in myolysis, fibrosis, and apoptosis in the CHF group $(\mathrm{p}<0.01)$. The protein expression of ADAMTS- 1 and Syndecan- 4 was dramatically up-regulated in the CHF group ( $p<0.01)$, and that of TIMP-3 was downregulated compared to those in the control group $(p<0.05)$. In addition, there was a positive correlation between the protein expression of ADAMTS- 1 and LVEDD in both groups $(r=0.804, p<0.01)$.

Conclusion Along with decreased TIMP-3, ADAMTS-1 is overexpressed in the ventricle of CHF rats and is associated with ventricular remodelling via cleaving the ectodomain of Syndecan-4. Therefore, ADAMTS-1 may provide a new therapeutic target in the prevention and treatment of ventricular remodelling in CHF.

\section{e0147 MECHANISM OF DIFFERENT ACTIVATORS OF PPAR $\alpha$ REGULATING PLASMINOGEN ACTIVATOR INHIBITOR-1 EXPRESSION}

doi:10.1136/hrt.2010.208967.147

${ }^{1}$ Chen Jing, ${ }^{2}{ }^{2}$ P Ping, ${ }^{1}$ Ren Xiangqun, ${ }^{1}$ Ding Jun, ${ }^{1}$ Huang Tongrui, ${ }^{1}$ Wang Chengzhang. ${ }^{1}$ Chinese Pla Peacehospital; ${ }^{2}$ Chinese Pla General Hospital

Objective To observe the effects of fenofibrate and linoleic acid which was the different activators of PPAR $\alpha$ on the expression of PAI-1 and PPAR $\alpha$ in HepG2 cells. To investigate the relationship and mechanisms between the promoter and expression of PAI-1 that how the fenofibrate and linoleic acid to act on PAI-1. And to observe if PPAR $\alpha$ involved in this procession.

Methods HepG2 cells were exposed to fenofibrate and linoleic acid in varying concentrations, RT-PCR was used to determine the mRNA expression of PAI-1 and PPAR $\alpha$. Several luciferase reporter gene recombinant plasmid containing different length sequences of human PAI-1 gene promoter from -804 to $+17 b p$ were constructed and transiently transfected into HepG2 cells. At the same time, co-transfected with PPAR $\alpha$-pSG5 expression plasmid, then different stimulating factors were added to induce the transfected cells. Transcriptional activity of PAI-1 was demonstrated by the measure of luciferase activity.

Results 1. Fenofibrate could remarkably decreased PAI-1 mRNA expression in HepG2 cells $(\mathrm{p}<0.05$ or $\mathrm{p}<0.01)$, but linoleic acid could significant increased PAI-1 mRNA level $(p<0.05$ or $p<0.01)$, and they were all in a concentration-dependent manner. 2. Fenofibrate and linoleic acid could raise the mRNA level of PPAR $\alpha(p<0.05$ or 
$p<0.01)$, which were also in a concentration-dependent way. 3. The PAI-1 transcriptional activity were significantly suppressed by fenofibrate $(p<0.05)$, but induced by linoleic acid $(p<0.01)$ in HepG2 cells transfected with PAI-pGL3 total length promoter constructs. 4. When co-transfected with PPAR $\alpha$-pSG5, fenofibrate could suppress the level of PAI-1 transcription further more $(p<0.05)$, while increased y linoleic acid $(\mathrm{p}<0.01)$. 5. The PAI-1 transcriptional activity were very inconsistent when transfected with the plasmid containing different length sequences of human PAI-1 gene promoter from -804 to $+17 \mathrm{bp}$.

Conclusions Fenofibrate and linoleic acid could increase the mRNA level of PPAR $\alpha$, and they regulate the synthesis of PAI-1 from transcriptional level, which was concerned with the activated of PPAR $\alpha$ by Fenofibrate and linoleic acid. The sequences that could regulate the expression of PAI-1 gene induced by fenofibrate might exist in the areas from -804 to -636 and -636 to -449 of PAI- 1 promoter and existed in the areas rom -804 to -636 and -449 to -276 induced by linoleic acid. The effects on expression of PAI-1 were very inconsistently, so there might be other mechanisms involved.

\section{e0148 CHANGES IN EXPRESSION OF ERK1/2, ANGIOTENSIN II RECEPTORS IN HIBERNATING MYOCARDIUM}

doi:10.1136/hrt.2010.208967.148

Zhu Hong, Li Dongye, Ding Qian. Affiliated Hospital of Xuzhou Medical College, Xuzhou, Jiangsu, China

Objective To investigate the variation and significance of ERK1/2, Angiotensin II subtype 1 receptor (AT1R) and Angiotensin II subtype 2 receptor $\left(\mathrm{AT}_{2} \mathrm{R}\right)$ in hibernating myocardium.

Methods 6 little domestic Chinese pigs were implanted a constrictor into the right coronary artery through femoral artery to make a immediate $50 \%-75 \%$ stenosis in the target artery. 1 month later after the operation, NTG ${ }^{99} \mathrm{TC}^{\mathrm{m}}$-MIBI SPECT (single photon emission $\mathrm{CT}$ ) was used to detect and locate hibernating myocardium before the animals were killed. Then verify the accuration of SPECT by observing the samples of hibernating myocardium (HM) under electron microscope. Finally assessing the variation of ERK1/ 2, p-ERK1/2 in normal myocardium and HM by western blot, AT1R and $\mathrm{AT}_{2} \mathrm{R}$ were localised by immunohistochemistry and quantified at protein level by western blot respectively.

Results 1 . The spatial distribution of AT1R showed no difference among NM and HM. AT1R were found in myocytes and vascular smooth muscle cells (VSMCs); $\mathrm{AT}_{2} \mathrm{R}$ were found only in myocytes in $\mathrm{NM}$, while in $\mathrm{HM} \mathrm{AT}_{2} \mathrm{R}$ could be found not only in myocytes but also in VSMCs. 2. Compared with NM, the relative amount of AT1R significently reduced in $\mathrm{HM}$ while $\mathrm{AT}_{2} \mathrm{R}$ significently increased in HM. 3. p-ERK1/2 were significantly increased in HM compared with NM.

Conclusion The changes of $\mathrm{AT} 1 \mathrm{R}$ and $\mathrm{AT}_{2} \mathrm{R}$ may help define the pathophysiological role of the angiotensin system in hibernatine myocardium.

\section{e0149 DIAGNOSTIC IMPLICATIONS OF TG/HDL-C AND PTX-3 IN DIAGNOSIS OF ACUTE CORONARY SYNDROME}

doi:10.1136/hrt.2010.208967.149

Zhang Li, Liu Qiming, Zhou Shenghua, Qin Haibin, Zhao Shuiping. The Department of Cardiology, The Second Xiangya Hospital, Central South University, Changsha, China

Objective To investigate the value of serum pentraxin-3 (PTX-3) together with triglyceride (TG)/high-density lipoprotein cholesterol (HDL-C) as a predictor risk factor for future acute coronary syndrome (ACS).

Methods Collected and analysed 171 cases of Department of Cardiology of the Second Xiangya Hospital of Central South
University in July 2008 December 2009 for elective coronary angiography patients, according to the situation on admission and coronary angiography, patients were divided into three groups: normal control subjects, stable angina pectoris and acute coronary syndrome group, all patients admitted to hospital were extracted fasting venous blood for measuring PTX-3 and lipids (TG, HDL-C) level in the next morning, and Statistically analysed, $\mathrm{p}<0.05$ was considered statistically significant.

Results The serum PTX-3 levels and the ratio of TG and HDL-C in patients with acute coronary syndrome $(6.39 \pm 3.01 \mathrm{ng} / \mathrm{ml}$; $2.38 \pm 2.00)$ were significantly increased than those in stable angina pectoris $(3.87 \pm 2.05 \mathrm{ng} / \mathrm{ml} ; 1.70 \pm 1.01)$ and normal control subjects $(2.90 \pm 1.94 \mathrm{ng} / \mathrm{ml}, 0.95 \pm 0.35), \mathrm{p}<0.05$.

Conclusions Increased serum PTX-3 and ratio of TG and HDL-C in patients are closely related with acute coronary syndrome, both increase accuracy of early diagnosis of acute coronary syndrome.

\section{Q0150 HIGH FREQUENCY OF PERI-STRUT LOW INTENSITY AREA ASSESSED BY OPTICAL COHERENCE TOMOGRAPHY AFTER POLYMER-BASED SIROLIMUS-ELUTING STENTS IMPLANTATION IN PORCINE MODEL}

doi:10.1136/hrt.2010.208967.150

Jia Haibo, Hou Jingbo, Wu Jian, Zhang Maomao, Qi Hai, Huang Xingtao, Liu Huiming, Hu Sining, Sun Rong, Zhang Shuo, Yu Bo. Department of Cardiology, Second Affiliated Hospital of Harbin Medical University, Key Laboratories of Education Ministry for Myocardial Ischaemia Mechanism and Treatment, Harbin, China

Objectives To assess the frequency of peri-strut low intensity area (PLIA) in polymeric and nonpolymeric drug-eluting stents by optical coherence tomography (OCT) in porcine model, to analyse the pathological changes in PLIA.

Setting Previous intravascular ultrasound studies showed that black holes were more commonly seen in sirolimus-eluting stent restenosis. Similar phenomenon (peri-strut low intensity area) was also frequently detected by OCT in DES follow-up. However, it is still largely unknown what triggers this uncommon response. Design and interventions: A total of 18 stents (BMS, $n=6$; polymer-free PES [PF-PES], $n=6$ and polymer-based SES [PB-SES], $n=6$ ) were implanted in six minipigs and OCT was performed at 28 days after stenting. Stented arteries were harvested after terminal OCT imaging for pathological analysis. PLIA was defined as a region around stent struts with a homogenous lower intensity appearance than surrounding tissue on OCT images without significant signal attenuation behind the area.

Results At 28 days, PLIA was more frequently observed around the PBSES struts compared with PFPES and BMS struts (75\% vs $33 \%$ vs $12 \%$, respectively, $\mathrm{p}<0.001)$. Both in DES and BMS group, stents with PLIA showed significantly greater neointimal thickness than stents without PLIA $(0.55 \pm 0.23 \mathrm{~mm}$ vs $0.13 \pm 0.08 \mathrm{~mm}, \mathrm{p}<0.001)$. Histological results showed the existence of fibrin deposition and small amount of inflammatory cells at the site of PLIA.

Conclusions PBSES showed a higher incidence of PLIA compared with BMS and PFPES. PLIA may be related to fibrin deposition and vessel chronic inflammatory response to stent.

\section{e0151 EXPRESSION OF IL-17 IN VIRAL INDUCED DILATED CARDIOMYOPATHY MICE}

doi:10.1136/hrt.2010.208967.151

Lin Song, Wu Weifeng. Department of Cardiology, Institute of Cardiovascular Diseases, The First Affiliated Hospital of Guangxi Medical University, Nanning, China

Objective To investigate Interleukin 17 (IL-17) levels in viral dilated cardiomyopathy (DCM) mice, aiming at effect of IL-17 in viral DCM. 